\title{
Some Simulation Results on the Computation of Delta of Path-Dependent Options Using a Discrete Version of Clark-Ocone Formula
}

\author{
Jirô Akahori, Takafumi Amaba and Kaori Okuma
}

\begin{abstract}
Department of Mathematical Sciences, Ritsumeikan University 1-1-1 Nojihigashi, Kusatsu-shi, Shiga, 525-8577, Japan

E-mail: \{jiro.akahori,capca0310,kaori.okuma\}@gmail.com
\end{abstract}

\begin{abstract}
In this paper, presented are some numerical computations of the hedging portfolio of plain, look-back and average options respectively, using a discrete version of Clark-Ocone formula proposed by the authors themselves in [1].
\end{abstract}

\section{Introduction}

1.1 A discrete version of Clark-Ocone formula Let $W$ be a Brownian motion and $X$ be a Wiener functional $^{1}$. Then it holds that

$$
X=E[X]+\int_{0}^{T} E\left[D_{s} X \mid \mathcal{F}_{s}\right] d W_{s},
$$

where $D_{s}$ means the Malliavin derivative. The formula (1) is known as Clark-Ocone formula $([2],[5],[6])$. In the context of mathematical finance, the formula gives an alternative description of the hedging portfolio in terms of Malliavin derivatives. However, explicit expressions of the Malliavin derivatives of a Wiener functional are not available in general, except for some special cases: see [7].

To overcome this difficulty, we have introduced a discrete version of (1), which is stated as follows. Define

$$
\mathcal{G}_{k}^{n}:=\sigma\left(\Delta W_{1}, \ldots, \Delta W_{k}\right)
$$

and for $F \in L^{2}\left(\mathscr{W}, \mathcal{G}_{n}^{n}, \nu\right)$, where $\mathscr{W}$ and $\nu$ denote the Wiener space and Wiener measure, respectively,

$$
F^{n}:=E\left[F \mid \mathcal{G}_{n}^{n}\right],
$$

where $n=2^{L}(L \in \mathbb{N}), \Delta t:=T / n, t_{k}:=k \cdot \Delta t, k=$ $0,1, \cdots, n$ and $\Delta W_{k}=W_{t_{k}}-W_{t_{k-1}}$.

Remark 1.1. Let $\mu^{n}$ be a probability measure on $\mathbb{R}^{n}$ defined by

$$
\mu^{n}(d x):=\frac{1}{(2 \pi \Delta t)^{\frac{n}{2}}} e^{-\frac{|x|^{2}}{2 \Delta t}} d x\left(x \in \mathbb{R}^{n}\right) .
$$

\footnotetext{
${ }^{1}$ For the definition of $\mathbb{D}^{1,2}$ in $\mathbb{D}^{1,2}$, see e.g. [4].
}

Then

$$
L^{2}\left(\mathscr{W}, \mathcal{G}_{n}^{n}, \nu\right) \simeq L^{2}\left(\mathbb{R}^{n}, \mathcal{B}\left(\mathbb{R}^{n}\right), \mu^{n}\right),
$$

and

$$
F \in \mathbb{D}^{1,2} \Rightarrow F^{n} \in \mathcal{W}^{1,2}\left(\mathbb{R}^{n}, \mathcal{B}\left(\mathbb{R}^{n}\right), \mu^{n}\right) .
$$

Here $\mathcal{W}^{1,2}\left(\mathbb{R}^{n}, \mathcal{B}\left(\mathbb{R}^{n}\right), \mu^{n}\right)$ consists of those functions whose first order derivatives in the distribution sense are in $L^{2}\left(\mathbb{R}^{n}, \mathcal{B}\left(\mathbb{R}^{n}\right), \mu^{n}\right)$, and its norm is given by

$$
\|X\|_{\mathcal{W}^{1,2}}^{2}=\|X\|_{L^{2}}^{2}+\sum_{l}\left\|\partial_{l} X\right\|^{2}
$$

where $\partial_{l} F^{n}$ means the partial derivative in $l$-th variable in the distribution sense.

The following is established in [1].

Theorem $1.2([1])$. If $F \in \mathbb{D}^{1,2}$, then

$E\left[\left(F-E[F]-\sum_{l=1}^{n} E\left[\partial_{l} F^{n} \mid \mathcal{G}_{l-1}^{n}\right] \Delta W_{l}\right)^{2}\right] \rightarrow 0(\Delta t \rightarrow 0)$.

The above theorem reads: we have a discrete version of Clark-Ocone formula as

$$
\begin{aligned}
F & \simeq E[F]+\sum_{l=1}^{n} E\left[\partial_{l} F^{n} \mid \mathcal{G}_{l-1}^{n}\right] \Delta W_{l} \\
& =E[F]+\int_{0}^{T} E\left[\partial_{l} F^{n} \mid \mathcal{G}_{l}^{n}\right] 1_{\left[\frac{l-1}{n}, \frac{l}{n}\right)}(s) d W_{s},
\end{aligned}
$$

which suggest the use of $E\left[\partial_{l} F^{n} \mid \mathcal{G}_{l}^{n}\right] 1_{\left[\frac{l-1}{n}, \frac{l}{n}\right)}(s)$ as an approximation of $E\left[D_{s} F \mid \mathcal{F}_{s}\right]$ (where for a set $A, 1_{A}(\cdot)$ is the indicator function). However, in most cases it is not easy to obtain $F^{n}$ out of a given $F$. In such a case, we may use an explicit function $X^{n} \in \mathcal{W}^{1,2}\left(\mathbb{R}^{n}, \mathcal{B}\left(\mathbb{R}^{n}\right), \mu^{n}\right)$ as a substitute. We established an error estimate for $X^{n}$ in [1]:

Theorem $1.3([1])$. For $X^{n} \in \mathcal{W}^{1,2}\left(\mathbb{R}^{n}, \mathcal{B}\left(\mathbb{R}^{n}\right), \mu^{n}\right)$, we have

$$
\begin{gathered}
E\left[\left(X^{n}-E\left[X^{n}\right]-\int_{0}^{T} E\left[\partial_{l} X^{n} \mid \mathcal{G}_{l}^{n}\right] 1_{\left[\frac{l-1}{n}, \frac{l}{n}\right)}(s) d W_{s}\right)^{2}\right] \\
\leq \Delta t\left\|X^{n}\right\|_{\mathcal{W}^{1,2}}^{2} .
\end{gathered}
$$


This means, a sequence $\left\{X^{n}\right\}$ with $E\left(F-X^{n}\right)^{2} \sim$ $O\left(n^{-1}\right)$ will provide an efficient/tractable "order $1 / 2$ " computation scheme for $E\left[D_{s} F \mid \mathcal{F}_{s}\right]$ if we could compute $E\left[\partial_{l} X^{n} \mid \mathcal{G}_{l}^{n}\right]$ properly. In fact, we have

$$
\begin{aligned}
& E\left[\left\{\int_{0}^{T}\left(E\left[D_{s} F \mid \mathcal{F}_{s}\right]-E\left[\partial_{l} X^{n} \mid \mathcal{G}_{l}^{n}\right] 1_{\left[\frac{l-1}{n}, \frac{l}{n}\right)}(s)\right) d W_{s}\right\}^{2}\right]^{\frac{1}{2}} \\
& \leq\left\|F-X^{n}\right\|+\left|E[F]-E\left[X^{n}\right]\right| \\
& \left.+\| X^{n}-E\left[X^{n}\right]-\int_{0}^{T} E\left[\partial_{l} X^{n} \mid \mathcal{G}_{l}^{n}\right] 1_{\left[\frac{l-1}{n}, \frac{l}{n}\right)}(s)\right) d W_{s} \| \\
& \leq 2\left\|F-X^{n}\right\| \\
& \left.+\| X^{n}-E\left[X^{n}\right]-\int_{0}^{T} E\left[\partial_{l} X^{n} \mid \mathcal{G}_{l}^{n}\right] 1_{\left[\frac{l-1}{n}, \frac{l}{n}\right)}(s)\right) d W_{s} \| \\
& \leq 2\left\|F-X^{n}\right\|+\sqrt{\frac{T}{n}} \sim O\left(n^{-\frac{1}{2}}\right) .
\end{aligned}
$$

In this paper we will construct $X^{n}$ out of a given $F$ as follows:

1. Let $\eta_{n}: \mathbb{R}^{n} \rightarrow C[0, T]$ be the embedding defined by $\left(x_{1}, \cdots, x_{n}\right) \mapsto\left\{\sum_{k=0}^{[n t / T]} x_{k}+\frac{n}{T}(t-\right.$ $\left.\left.\left[\frac{n t}{T}\right]\right) x_{\left[\frac{n t}{T}\right]+1}\right\}$, where $C[0, T]$ denotes the space of piecewise linear paths and $x_{0}$ is constant.

2. We set $X^{n}=F \circ \eta_{n}$, where we $\operatorname{regard} F$ as a function of $C[0, T]$ in which the Wiener space is embedded.

(The error estimate of $E\left(F-X^{n}\right)^{2}$ is left behind.)

\subsection{The computation of delta under Black- Scholes economy}

In the present paper, we will compute the "delta" of some path-dependent options under the Black-Scholes assumptions using the above discrete Clark-Ocone scheme.

Assume that the price $S$ of risky asset follows a geometric Brownian motion:

$$
S_{t}=S_{0} \exp \left(\sigma W_{t}-\frac{\sigma^{2}}{2} t\right), t>0
$$

where $S_{0}>0$ is a given initial data, $\sigma$ is the volatility and $W$ is a one dimensional Brownian motion under the risk neutral measure. Here we assume that the interest rate is zero for simplicity.

The pay-off of a path-dependent option written on the risky asset is described as a functional of $S$, which is also a functional of $W$ since $S$ itself is a functional of $W$. Let $X \in \mathbb{D}^{1,2}$ be the corresponding Wiener functional of a path-dependent option. The hedging strategy $\theta$, or in other words, the "delta" (see(Hull)) is given by

$$
\theta_{s}=\frac{E\left[D_{s} X \mid \mathcal{F}_{s}\right]}{\sigma S_{s}}
$$

since $d S_{s}=\sigma S_{s} d W_{s}$, or more precisely

$$
X=E[X]+\int_{0}^{T} \frac{E\left[D_{s} X \mid \mathcal{F}_{s}\right]}{\sigma S_{s}} d S_{s} .
$$

In this paper we will deal with two typical examples of path-dependent options:

1. Look back option: its pay-off is given by $\max _{0 \leq u \leq T} S_{u}-S_{T}$. It is approximated by $X^{n}:=$ $\max _{0<k<n} e^{\sigma \sum_{i=1}^{k} \Delta W_{i}-\frac{\sigma^{2}}{2 n} k T}-e^{\sigma \sum_{i=1}^{n} \Delta W_{i}-\frac{\sigma^{2}}{2} T}$. Since we know a closed form delta formula for this option, we can consider the result as a benchmark.

2. Asian option (average option): the payoff is given by $\left(\frac{1}{T} \int_{0}^{T} S_{s} d s-K\right)^{+}$(where $\left.A^{+}=\max (A, 0)\right)$, where $K$ is a strike price. It is approximated by $\left(\frac{S_{0}}{T}\left\{\int_{0}^{t_{1}} e^{\sigma \frac{n}{T} s \Delta W_{1}-\frac{1}{2} \sigma^{2} s} d s+\right.\right.$ $\left.\sum_{l=2}^{n} \int_{t_{l-1}}^{t_{l}} e^{\sum_{k=1}^{l-1} \sigma \Delta W_{k}+\sigma \frac{n}{T}\left(s-t_{l-1}\right) \Delta W_{l}-\frac{1}{2} \sigma^{2} s}\right\} \quad-$ $K)^{+}$. If we used Yor's formula, it would be possible to obtain a closed form of the delta, but we will not work on this since it is too complicated.

Other that these, as a benchmark of our framework, we will present a simulation results for the plain call option, pay-off of which is $F=\left(S_{T}-K\right)^{+}$. In this case we do not have to approximate the pay-off since

$$
F^{n}=E\left[F \mid \mathcal{G}_{n}^{n}\right]=\left(S_{0} e^{\sigma \sum_{i=1}^{n} \Delta W_{i}-\frac{\sigma^{2}}{2} T}-K\right)^{+}=F .
$$

Remark 1.4. In [3], Leitz-Martini introduced another discrete version of (1) in the Bernoulli random walk setting. The random walk has a martingale representation property and therefore a perfect hedging strategy will be given by the discrete Clark-Ocone formula. On the other hand, embedding the Bernoulli setting to the standard continuous time framework is quite tedious. The priority of our methods lies in the tractability of the embedding, its error estimate, and specific computations.

\section{Numerical Results}

In this paper, we will give the simulation results of the approximating values of the hedging portfolio of the three path-dependent options together with the benchmark result of the plain call option, as described above.

\subsection{The plain vanilla case: a benchmark}

First, we will give the simulation results of the plain option, and draw a comparison between them and a theoretical value obtained by Black-Scholes formula (4).

$$
\theta_{0}:=\text { BS hedging }=N(d),
$$

where $d=\frac{\log \frac{S_{0}}{K}+\frac{\sigma^{2} T}{2}}{\sigma \sqrt{T}}(\operatorname{see}[8])$. Let $S_{0}$ denote the initial stock price and $K, \sigma$ and $T$ denote the strike, the volatility and the maturity respectively. We will consider plane call option with the initial stock price We set $S_{0}=100$, strike $K=120$, maturity $T=0.5$ and volatility $\sigma=0.3$. The theoretical value obtained by Black-Scholes formula (4) is 0.2256030 . 
On the other hand, the hedging portfolio of $X^{(n)}\left(\Delta W_{1}, \cdots, \Delta W_{n}\right)=\left(S_{0} \exp \left(\sigma \sum_{k=1}^{n} \Delta W_{k}-\right.\right.$ $\left.\left.\frac{\sigma^{2}}{2} t_{n}\right)-K\right)^{+}$at time $t_{i}$ obtained by Theorem 1.2 is the following:

$$
\begin{aligned}
\theta_{t_{i-1}}^{(n)} & :=E\left[\partial_{i} X^{(n)} \mid \mathcal{G}_{i-1}^{n}\right] / \sigma S_{t_{i-1}} \\
& =E\left[1_{[K, \infty)}\left(S_{0} \exp \left(\sigma \sum_{k=1}^{n} \Delta W_{k}-\frac{\sigma^{2}}{2} t_{n}\right)\right)\right. \\
& \left.\sigma S_{0} \exp \left(\sigma \sum_{k=1}^{n} \Delta W_{k}-\frac{\sigma^{2}}{2} t_{n}\right) \mid \mathcal{G}_{i-1}^{n}\right] / \sigma S_{t_{i-1}}
\end{aligned}
$$

In particular, if $i=1$, then

$$
\begin{aligned}
& \theta_{0}^{(n)}= E\left[\partial_{1} X^{(n)}\right] / \sigma S_{0} \\
&=E\left[1_{[K, \infty)}\left(S_{0} \exp \left(\sigma \sum_{k=1}^{n} \Delta W_{k}-\frac{\sigma^{2}}{2} t_{n}\right)\right)\right. \\
&\left.\quad \exp \left(\sigma \sum_{k=1}^{n} \Delta W_{k}-\frac{\sigma^{2}}{2} t_{n}\right)\right] .
\end{aligned}
$$

Though we can calculate (6) directly using Gauss kernel just as the theoretical value (4), we use a Monte Carlo method aiming that it gives, as a benchmark, information of the accuracy and the speed (and the mixture) of our framework. To accomplish the task, we generate $n$ (the number of the time step discretization $\times N$ (the number of the sample paths for the Monte-Carlo simulation) Gaussian (quasi-)random numbers with mean 0 and variance $\frac{T}{n}$.

We set $N=1000000$ and $n=256$ in the simulation of Fig. 1. The values and the errors are indicated in Table 1. It is very accurate and they took just 43 seconds respectively. This accuracy is almost always attained.

\begin{tabular}{|l|c|}
\hline value & error(\%) \\
\hline 0.225844 & 0.106824821 \\
\hline 0.225435 & -0.074467095 \\
\hline 0.22504 & -0.249553419 \\
\hline 0.225616 & 0.005762335 \\
\hline 0.225743 & 0.062055912 \\
\hline
\end{tabular}

Table 1: Simulation results of $\theta_{1}^{(256)}=E\left[\partial_{1} X^{(256)}\right] / \sigma S_{0}$ in the case of the plain option.

\subsection{Look-back option}

As we have written, we use

$$
\begin{aligned}
& X^{(n)}\left(\Delta W_{1}, \ldots, \Delta W_{n}\right) \\
&=S_{0} \max \left(1, \max _{1 \leq l \leq n} e^{\sum_{k=1}^{l} \sigma \Delta W_{k}-\frac{1}{2} \sigma^{2} t_{l}}\right) \\
& \quad-S_{0} e^{\sum_{k=1}^{n} \sigma \Delta W_{k}-\frac{1}{2} \sigma^{2} t_{n}} .
\end{aligned}
$$

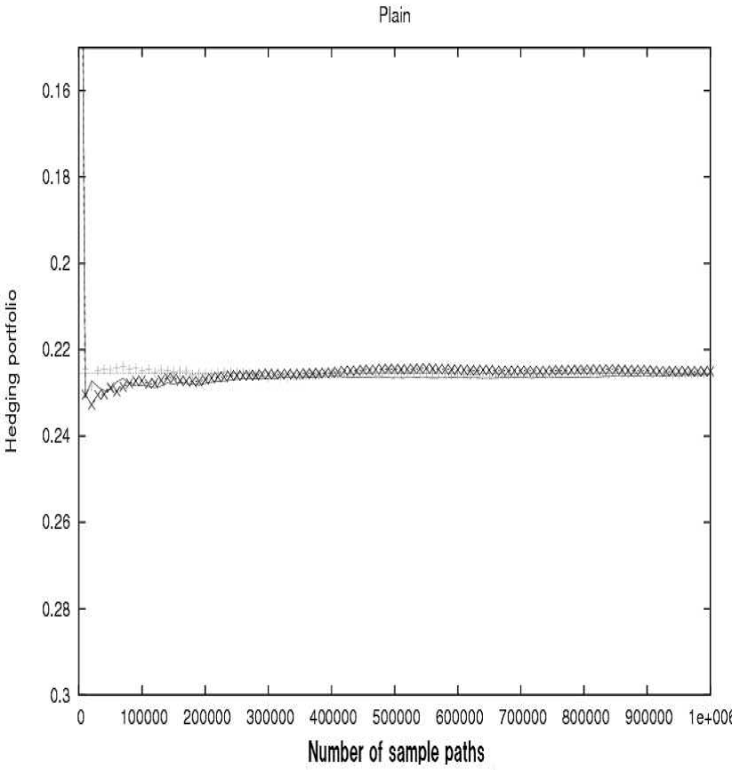

Fig. 1: Simulation results of $\theta_{1}^{(256)}=E\left[\partial_{1} X^{(256)}\right] / \sigma S_{0}$ in the case of the plain option.

A closed form of the delta is known in this case (see e.g. [8]) as

$$
\begin{aligned}
& \frac{\sigma \sqrt{T-t}}{\sqrt{2 \pi}} \frac{Y(t)}{S(t)} e^{-\frac{\delta_{-}\left(T-t, \frac{S(t)}{Y(t)}\right)^{2}}{2}} \\
&+\left(\log \frac{S(t)}{Y(t)}+\frac{\sigma(T-t)}{2}\right) N\left(-\delta_{-}\left(T-t, \frac{Y(t)}{S(t)}\right)\right) \\
&+2 N\left(\delta_{+}\left(T-t, \frac{S(t)}{Y(t)}\right)\right)-1
\end{aligned}
$$

where $S(t)=S_{0} e^{\sigma W_{t}-\frac{\sigma^{2} t}{2}}, Y(t)=\max _{0 \leq u \leq t} S(u)$, $\delta_{ \pm}(\tau, z)=\frac{1}{\sigma \sqrt{\tau}}\left(\log z \pm \frac{\sigma^{2} \tau}{2}\right)$. At $\mathrm{t}=0$,

$$
\begin{aligned}
\frac{\sigma \sqrt{T}}{\sqrt{2 \pi}} e^{-\frac{\delta_{-}(T, 1)}{2}{ }^{2}}+\frac{\sigma^{2} T}{2} N( & \left.-\delta_{-}(T, 1)\right) \\
& +2 N\left(\delta_{+}(T, 1)\right)-1,
\end{aligned}
$$

where $\delta_{ \pm}(T, 1)= \pm \frac{\sigma \sqrt{T}}{2}$.

We will calculate the price of a look back call option with the initial stock price $S_{0}=100$, maturity $T=0.5$ and volatility $\sigma=0.3$. The theoretical value obtained by (8) is 0.1808241 .

On the other hand, the hedging portfolio of $X^{(n)}\left(\Delta W_{1}, \ldots, \Delta W_{n}\right) \quad$ $S_{0} \max \left(1, \max _{1 \leq l \leq n} e^{\sum_{k=1}^{l} \sigma \Delta W_{k}-\frac{1}{2} \sigma^{2} t_{l}}\right)$ $S_{0} e^{\sum_{k=1}^{n} \sigma \Delta W_{k}-\frac{1}{2} \sigma^{2} t_{n}}$ at time 0 obtained by our 


\begin{tabular}{|l|c|}
\hline value & error(\%) \\
\hline 0.131884 & -27.06503171 \\
\hline 0.131886 & -27.06392566 \\
\hline 0.1316 & -27.22209042 \\
\hline 0.131784 & -27.12033407 \\
\hline 0.131715 & -27.1584927 \\
\hline
\end{tabular}

Table 2: Simulation results of $\theta_{1}^{(256)}=E\left[\partial_{1} X^{(256)}\right] / \sigma S_{0}$ in the case of look back option.

DCO formula is the following:

$$
\begin{aligned}
& \theta_{0}^{(n)}:= E\left[\partial_{1} X^{(n)}\right] / S_{0} \sigma \\
&=E\left[1_{[1, \infty)}\left(\max _{1 \leq l \leq n} e^{\sum_{k=1}^{l} \sigma \Delta W_{k}-\frac{1}{2} \sigma^{2} t_{l}}\right)\right. \\
&\left.\max _{1 \leq l \leq n} e^{\sum_{k=1}^{l} \sigma \Delta W_{k}-\frac{1}{2} \sigma^{2} t_{l}}-e^{\sigma \sum_{k=1}^{n} \Delta W_{k}-\frac{\sigma^{2}}{2} t_{n}}\right] .
\end{aligned}
$$

We set $N=1000000$ and $n=256$ in the simulation of Fig. 2. It looks the simulation value converges to a wrong one. Equation (9) implies that if we increase the number of partitions, we will get a value closer to the theoretical value. How closer the values are (as the number increases) are indicated in Fig. 3 and Table 3, by which we may conclude that the simulation with 131072 partitions is fine. We set $N=1000$ and $n=131072$ and the results of five simulations are given in Fig. 4 and Table 4. Each simulation took 26 seconds.

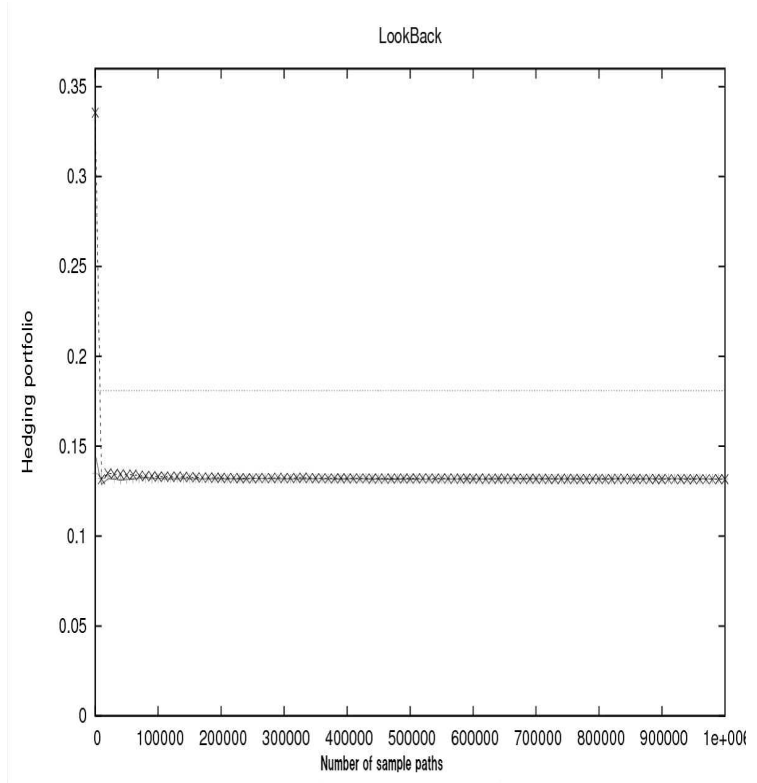

Fig. 2: Simulation results of $\theta_{1}^{(256)}=E\left[\partial_{1} X^{(256)}\right] / \sigma S_{0}$ in the case of look back option.

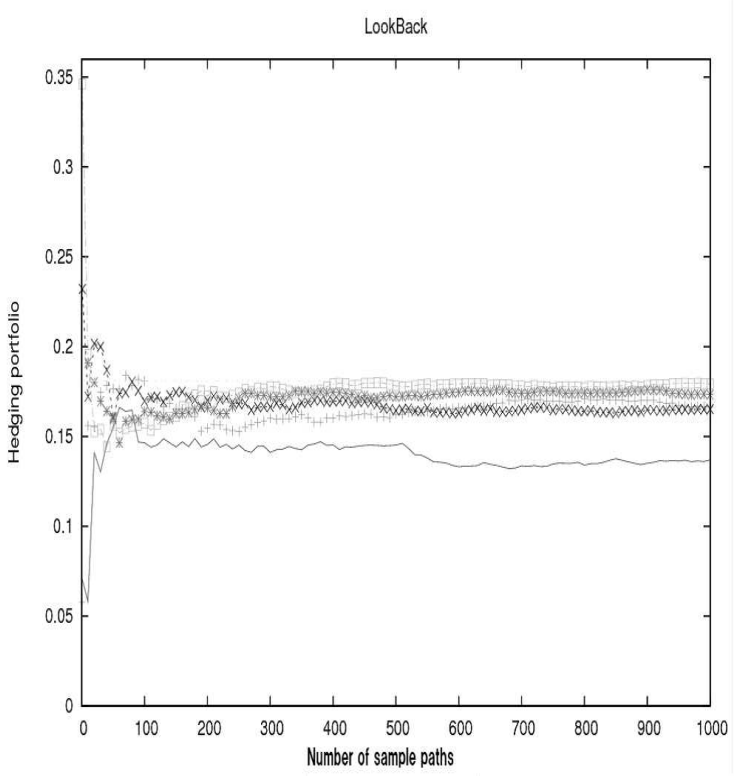

Fig. 3: Simulation results of $\theta_{1}^{(n)}=E\left[\partial_{1} X^{(n)}\right] / \sigma S_{0}$, $n=512,2048,8192,32768,131072$, in the case of look back option.

\begin{tabular}{|l|c|r|}
\hline partitions & value & error(\%) \\
\hline 512 & 0.13698 & -24.24682329 \\
\hline 2048 & 0.166283 & -8.041571892 \\
\hline 8192 & 0.165227 & -8.625564845 \\
\hline 32768 & 0.173249 & -4.189209292 \\
\hline 131072 & 0.1792656 & -0.86221914 \\
\hline
\end{tabular}

Table 3: Simulation results of $\theta_{1}^{(n)}=E\left[\partial_{1} X^{(n)}\right] / \sigma S_{0}$, $n=512,2048,8192,32768,131072$, with $N=1$ million in the case of look back option.

\begin{tabular}{|l|c|}
\hline value & error(\%) \\
\hline 0.178158 & -1.474416297 \\
\hline 0.181798 & 0.538589712 \\
\hline 0.179054 & -0.978907126 \\
\hline 0.175995 & -2.670606407 \\
\hline 0.177141 & -2.036841328 \\
\hline
\end{tabular}

Table 4: Simulation results of $\theta_{1}^{(131072)}=$ $E\left[\partial_{1} X^{(131072)}\right] / \sigma S_{0}$ in the case of look back option. 


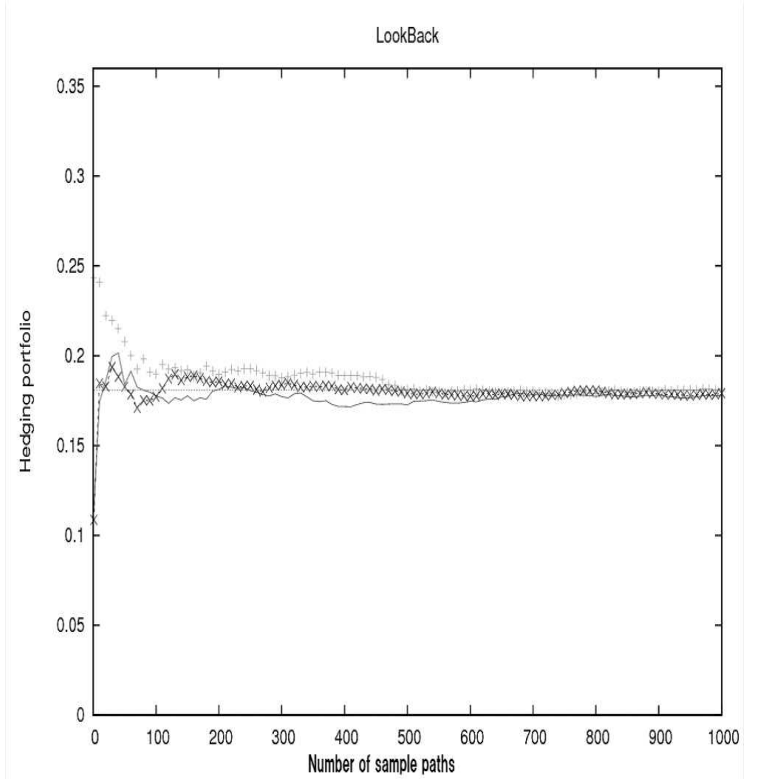

Fig. 4: Simulation results of $\theta_{1}^{(131072)}=$ $E\left[\partial_{1} X^{(131072)}\right] / \sigma S_{0}$ in the case of look back option.

\subsection{Asian option}

As we have described, we use

$$
\begin{gathered}
X^{(n)}\left(\Delta W_{1}, \ldots, \Delta W_{n}\right) \\
=\left(\frac { S _ { 0 } } { T } \left\{\int_{0}^{t_{1}} e^{\sigma \frac{n}{T} s \Delta W_{1}-\frac{1}{2} \sigma^{2} s} d s\right.\right. \\
\left.\left.+\sum_{l=2}^{n} \int_{t_{l-1}}^{t_{l}} e^{\sum_{k=1}^{l-1} \sigma \Delta W_{k}+\sigma \frac{n}{T}\left(s-t_{l-1}\right) \Delta W_{l}-\frac{1}{2} \sigma^{2} s} d s\right\}-K\right)^{+} \\
=\left(\frac { S _ { 0 } } { T } \left\{\int_{0}^{t_{1}} e^{\sigma \frac{n}{T} s \Delta W_{1}-\frac{1}{2} \sigma^{2} s} d s\right.\right. \\
\left.\left.+\sum_{l=2}^{n} e^{\sum_{k=1}^{l-1} \sigma \Delta W_{k}} \int_{t_{l-1}}^{t_{l}} e^{\sigma \frac{n}{T}\left(s-t_{l-1}\right) \Delta W_{l}-\frac{1}{2} \sigma^{2} s} d s\right\}-K\right)^{+} .
\end{gathered}
$$

We can calculate the differentiation in this case as

$$
\begin{gathered}
\theta_{0}^{(n)}=E\left[\partial_{1} X^{(n)}\right] / \sigma S_{0} \\
=E\left[1 _ { [ K , \infty ) } \left(\frac { S _ { 0 } } { T } \left\{\int_{0}^{t_{1}} e^{\sigma \frac{n}{T} s \Delta W_{1}-\frac{1}{2} \sigma^{2} s} d s\right.\right.\right. \\
\left.\left.+\sum_{l=2}^{n} e^{\sum_{k=1}^{l-1} \sigma \Delta W_{k}} \int_{t_{l-1}}^{t_{l}} e^{\sigma \frac{n}{T}\left(s-t_{l-1}\right) \Delta W_{l}-\frac{1}{2} \sigma^{2} s} d s\right\}\right) \\
\quad \times \frac{1}{T}\left\{\int_{0}^{t_{1}} \frac{n}{T} s e^{\sigma \frac{n}{T} s \Delta W_{1}-\frac{1}{2} \sigma^{2} s} d s\right. \\
\left.\left.+\sum_{l=2}^{n} e^{\sum_{k=1}^{l-1} \sigma \Delta W_{k}} \int_{t_{l-1}}^{t_{l}} e^{\sigma \frac{n}{T}\left(s-t_{l-1}\right) \Delta W_{l}-\frac{1}{2} \sigma^{2} s} d s\right\}\right]
\end{gathered}
$$

We will consider the price of an Asian call option with the initial stock price $S_{0}=100$, strike $K=120$, maturity $T=0.5$ and volatility $\sigma=0.3$. We rely again on the Monte Carlo simulation as we have done for the previous cases. Fig. 5 and Table 5 show how the simulated

\begin{tabular}{|l|c|}
\hline partitions & value \\
\hline 16 & 0.076709 \\
\hline 64 & 0.078467 \\
\hline 256 & 0.079045 \\
\hline 1024 & 0.078402 \\
\hline 4096 & 0.078957 \\
\hline
\end{tabular}

Table 5: Simulation results of $\theta_{1}^{(n)}=E\left[\partial_{1} X^{(n)}\right] / \sigma S_{0}$, $n=16,64,256,1024,4096$, with $N=1$ million

values converge as the number of partitions increases. They imply that a simulation with 64 partitions suffice. We set $N=1000000$ and $n=64$ in the simulations of Fig. 6 and Table 6. They took 199 seconds.

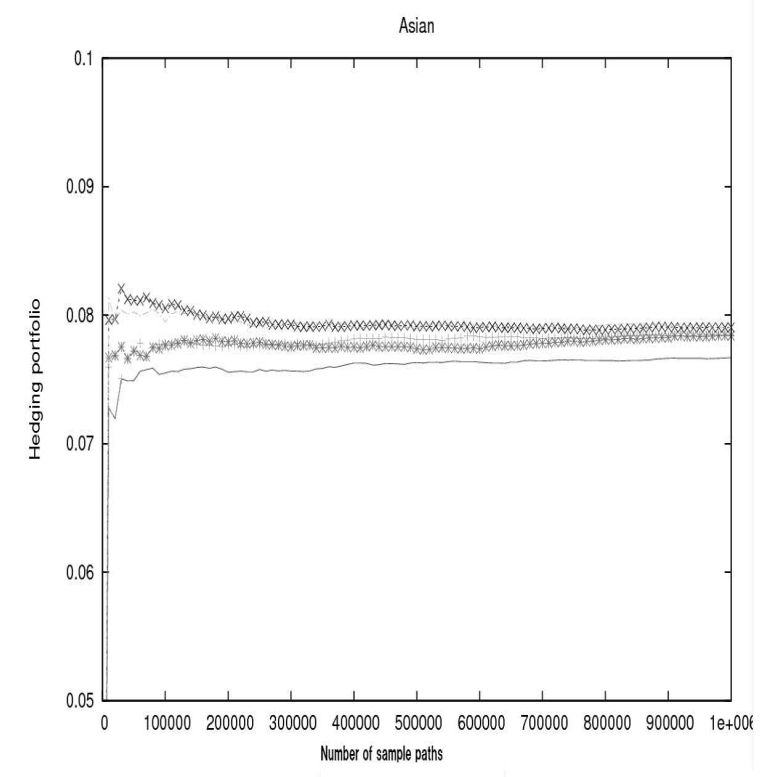

Fig. 5: Simulation results of $\theta_{1}^{(n)}=E\left[\partial_{1} X^{(n)}\right] / \sigma S_{0}$, $n=16,64,256,1024,4096$, with $N=1$ million

\section{Conclusion}

We have used a discrete version of Clark-Ocone formula as a tool for computing the delta, hedging portfolio, of path-dependent options under the Black-Scholes economy. The simulation results imply that

- For the look back case: the stability of Monte Carlo simulation is not bad, but the convergence in respect of time discretization does not seem nice.

- For the Asian: the stability of Monte Carlo simulation is not so good, but the convergence in respect of time discretization is not bad.

We have only considered the Geometric Brownian case. If the underlying price process is given by a solution 


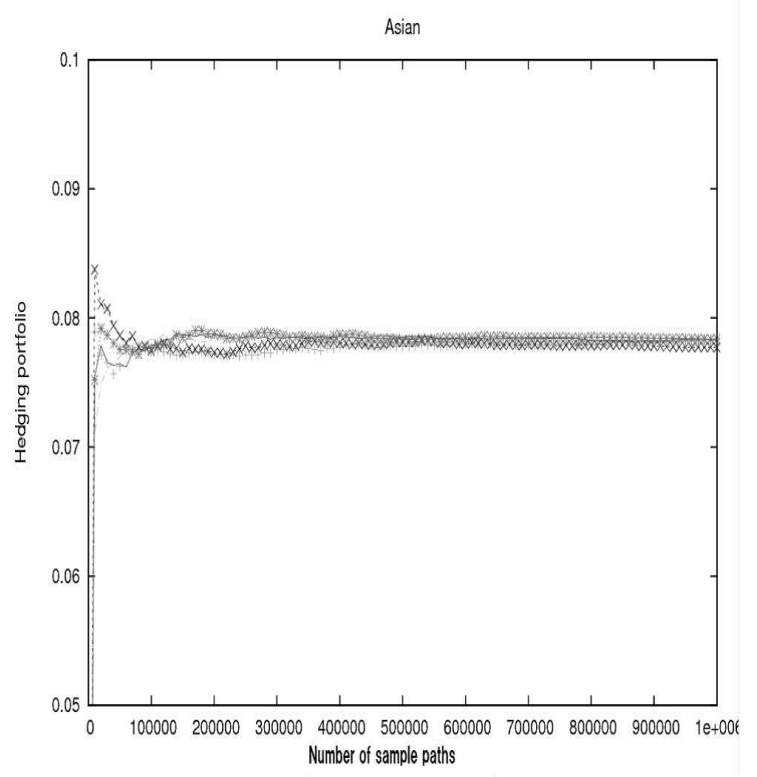

Fig. 6: the simulation results of $\theta_{1}^{(64)}=$ $E\left[\partial_{1} X^{(64)}\right] / \sigma S_{0}$,with $N=1$ million

\begin{tabular}{|l|}
\hline value \\
\hline 0.078259 \\
\hline 0.078397 \\
\hline 0.077716 \\
\hline 0.078366 \\
\hline 0.077904 \\
\hline
\end{tabular}

Table 6: Simulation results of $\theta_{1}^{(64)}=E\left[\partial_{1} X^{(64)}\right] / \sigma S_{0}$, with $N=1$ million

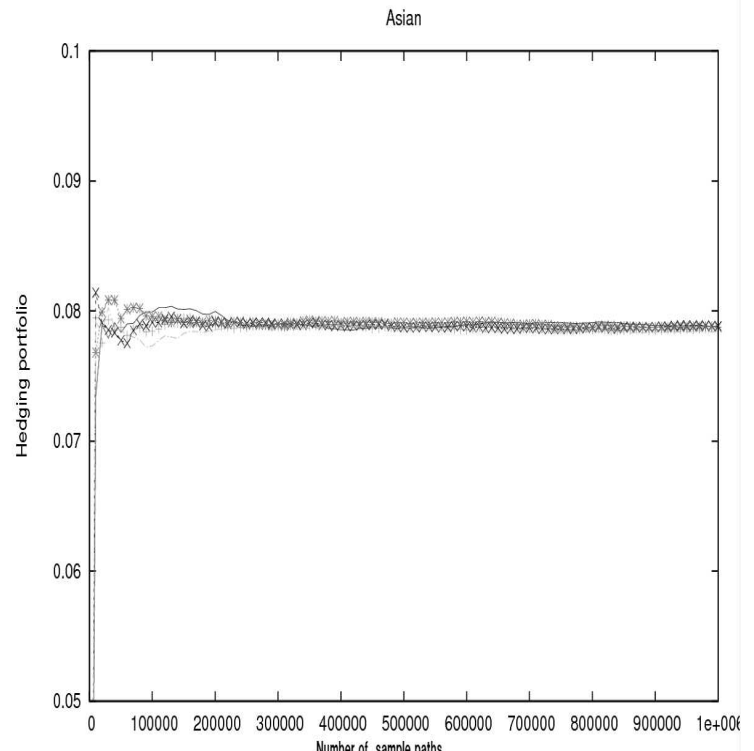

Fig. 7: Simulation results of $\theta_{1}^{(256)}=E\left[\partial_{1} X^{(256)}\right] / \sigma S_{0}$, with $N=1$ million

\begin{tabular}{|l|}
\hline value \\
\hline 0.078836 \\
\hline 0.078730 \\
\hline 0.078845 \\
\hline 0.078774 \\
\hline 0.078443 \\
\hline
\end{tabular}

Table 7: Simulation results of $\theta_{1}^{(256)}=$ $E\left[\partial_{1} X^{(256)}\right] / \sigma S_{0}$, with $N=1$ million

to a more complicated stochastic differential equation, we need another tractable scheme to obtain $X^{n}$. Also, some error estimated should be established.

\section{References}

[1] Akahori, J., Amaba, T. And Okuma, K.(2011): "A discrete version of Clark-Ocone formula and its application to the computation of delta of path-dependent options", preprint.

[2] Clark, J.M.C.(1970): "The representation of functionals of Brownian motion by stochastic integral", Ann.Math.Statist.41,1282-1295.

[3] Leitz-Martini, M.(2000) "A discrete ClarkOcone formula", Maphysto Research Report No 29, http://www.maphysto.dk/publications/MPS$\mathrm{RR} / 2000 / 29 . p d f$

[4] Nualart, D. (1995) The Malliavin Calculus and Related Topics Springer.

[5] Ocone, D. (1984): "Malliavin's calculus and stochastic integral representations of functionals of diffusion processes", Stochastics 12, no. 3-4, 161185.

[6] Ocone, D., Karatzas, I. (1991) :"A generalized Clark representation formula, with application to optimal portfolios", Stochastics Rep. 34, no. 3-4, 187-220.

[7] Renaud,J.F. and RÉmillard,B. (2007): "Explicit martingale representations for Brownian functionals and applications to option hedging" Stochastic Analysis and Applications, 25 , 810820 .

[8] Shreve,S.E., "Stochastic Calculus for Finance II Continuous-Time Models" 118, 308-320, 335 